\title{
Lubrication Theory Analysis of the Permeability of Rough-Walled Fractures
}

\author{
R.W. Zimmerman, S. Kumar, and G. S. Bodvarsson \\ Earth Sciences Division \\ Lawrence Berkeley Laboratory \\ University of Califorina \\ Berkeley, California 94720
}

January 1991

DISCLAIMER

\begin{abstract}
This report was prtpared as an account of work sponsored by an agency of the United States Government. Neither the United States Government nor any agency thereol, nor any of their employees, makes any warranty, express or implied, or assumes any legal liability or responsibility for the accuracy, completeness, or uselulness of any information, apparatus, product. or process disclosed, or represents that its use would not infringe privately owned rights. Reference herein to any specific commercial product, process, or service by trade name, trademark, manufacturer, or otherwise does not necessarily constitute or imply its endorsement, recommendation, or favoring by the United States Government or any agency thereor. The views and opinions of authors expressed bercin do not necessarily state or reflect those of the United States Government or any egency thereof.
\end{abstract}

This work was carricd out under U.S. Deparment of Encrgy Contract No. DE-AC03-76SF00098, atmin. istered by the DOE Nevada Orfice, in cooperation with the U.S. Geological Survey, Denver. 


\title{
LUBRICATION THEORY ANALYSIS OF THE PERMEABILITY OF ROUGH.WALLED FRACTURES
}

\author{
R. W. ZIMMERMAN*, S. KUMAR, and G. S. BODVARSSON
}

\author{
Earth Sciences Division \\ Lawrence Berkeley Laboratory \\ University of California \\ Berkeley, CA 94720 \\ *(415) 486-7106
}

\begin{abstract}
Lubrication theory is used to study the permeability of rough-walled rock fractures. Two idealized models of a fracture, in which the roughness follows a sinusoidal or a sawtooth variation, are studied in detail. The results are similar to those found previously from numerical analysis of fractures with random aperture distributions, in that the ratio of the hydraulic aperture depends mainly on the mean and the standard deviation of the aperture distribution. Higher-order approximations to the Navier-Stokes equations are then studied, and it is concluded that the validity of the lubrication approximation requires the fracture walls to be smooth over lengths on the order of one standard deviation of the apenure.
\end{abstract}




\section{INTRODUCTION}

The flow of a fluid between the rough surfaces of a rock fracture is very complex, due to the tortuous paths followed by the fluid paricies. Exact analytical modeling of these flows is made difficult by the irregular geometry of rock fracture surfaces, while full three-dimensional numerical simulations of these flows are as yet still impractical. To overcome the difficulties of working with the three-dimensional Navier-Stokes equations, the simpler Reynolds Iubrication equation has sometimes been used to model flow in fractures. ${ }^{1,2}$ This paper focuses on two aspects of lubrication theory. First, lubrication theory is applied to two simplified aperture profiles, sinusoidal and "sautooth", and analytical expressions are found for the permeabilities. These results are then compared with numerical results obtained by solving the lubrication equation for fractures with "random" surfaces. ${ }^{2,3}$ Secondly, the validity of the lubrication equations for modeling flow in rough fractures is studied by examining higher-order perturbaiion solutions, as well as numerical solutions, to the Navier-Stokes equations for flow in fractures with sinusoidally-varying apertures.

\section{LUBRICATION THEORY MODEL}

The flow of a Newtonian fluid through a rock fracture is governed by the NavierStokes equations of fluid mechanics. ${ }^{4,5}$ These are a set of three coupled, nonlinear partial differential equations, and only for very simplified cases can they be solved analytically. One such case is that of flow under a uniform pressure gradient in the channel between two parallel, smooth surfaces. For this problem only one component of the velocity vector is nonzero, and the equations simplify greatly. The result is the wellknown parabolic velocity profile, with the volumetric flow rate per unit width perpendicular to the direction of flow given by $Q=d^{3} \Delta P / 12 \mu \mathrm{L}$, (the so-called "cubic law"), in which $d$ is the aperture of the channel, $\mu$ is the dynamic viscosity of the fluid, anc $\triangle \mathrm{P} / \mathrm{L}$ is the magnitude of the pressure gradient. ${ }^{6}$ If the aperture is not constant along 
the channel, the equations cannot be sclved analytically, and one must resort to approximate methods of some sort.

Under certain geometric and kinematic conditions which usually are assumed to hold for rock fractures, the Navier-Stokes equations can be reduced (locally) to the simpler Reynolds equation. One requirement for the Reynolds equation to be valid is that viscous forces dominate the inertial forces. A quantitative statement of this criterion is that the "reduced Reynolds number", $\mathrm{Re}^{*}$, be very small, i.e., ${ }^{4}$

$$
\operatorname{Re}^{*}=\frac{\mathrm{pUd}_{\mathrm{m}}^{2}}{\mu \Lambda} \ll 1,
$$

where $\rho$ is the fluid density, $U$ is the average velocity along the fracture, $d_{m}$ is the mean aperture, and $\Lambda$ is some characteristic length of the fracture in the direction of the flow. There are also geometric conditions which specify that, in some sense, the aperture does not change too abruptly; these geometric conditions will be examined more closely below. If these dynamical and gcometric conditions hold, the flow can be described by the Reynolds equation:

$$
\frac{\partial}{\partial x}\left[d^{3}(x, y) \frac{\partial P}{\partial x}\right]+\frac{\partial}{\partial y}\left[d^{3}(x, y) \frac{\partial P}{\partial y}\right]=0
$$

where $(x, y)$ are orthogonal coordinates in the plane of the fracture, and $d(x, y)$ is the local aperture of the fracture. Eq. (2) is a single, linear partial differential equation that describes the pressure field in the fracture plane. The volumetric flow of liquid is then related to the pressure by 


$$
\vec{Q}=\frac{-d^{3}(x, y)}{12 \mu} \nabla P
$$

Milne-Thomson ${ }^{7}$ derived the Reynolds equation from the Navier-Stokes equations through an order-of-magnitude analysis to allow the elimination of certain terms. Walsh ${ }^{1}$ derived (2) by merely assuming that the cubic law holds locally at each point in the fracture, and then invoking the principle of conservation of mass. Since the conditions under which eq. (2) is valid usually hold for the flow of lubricants that are used to reduce friction and wear in moving machinery parts, this is sometimes known as the lubrication approximation.

\section{EFFECT OF APERTURE VARIATIONS}

Brown ${ }^{2.8}$ solved eq. (2) numerically for a fracture with a randomly-generated fractal apenure distribution, and plotted the fracture permeability as a function of the ratio of the mean aperture $d_{m}$ to its standard deviation $\sigma$. The permeability was quantified in terms of the hydraulic aperture $d_{h}$, which is that value of $d$ that would allow the flow rate to exactly satisfy the cubic law. The permeability was found to decrease as the standard deviation of the apenture increased (for fixed mean aperture), and was also found to be insensitive to the fractal dimension of the fracture surface. These results are remarkably similar to those of Patir and Cheng, ${ }^{3}$ who performed a similar analysis of lubrication flow between surfaces whose profiles ubeyed a Gaussian distribution with linearly-decreasing auto-correlation functions. Fig. 1 shows the normalized permeabilities, in the form of $\left(\mathrm{d}_{\mathrm{h}} / \mathrm{d}_{\mathrm{m}}\right)^{3}$, computed by Brown ${ }^{8}$ for a surface fractal dimension of $\mathrm{D}=2.5$, along with the calculations of Patir and Cheng. ${ }^{3}$ Each one of Brown's data points represents the mean of ten different realizations. The solid curve, which was found by Patir and Cheng to provide a reasonable fit to their numerical results, can be expressed by 


$$
\left[\frac{d_{h}}{d_{m}}\right]^{3}=1-0.90 e^{-0.56 d_{m} / \sigma} .
$$

The similarity between the results of $\mathrm{BrC}^{\mathrm{r}} \mathrm{n}^{8}$ and Patir and Cheng ${ }^{3}$ suggests some universal (approximate) validity of the correlation (4) between the dimensionless parameters $d_{h} / d_{m}$ and $d_{m} / \sigma$ that is otherwise independent of the statistics of the aper. ture distribution. (The results found by Brown ${ }^{8}$ for different fracial dimensions very nearly coincided. The data shown in Fig. 1 were for the case in which "conservation of volume" was imposed on the fracture during its deformation. The details of this constraint are not relevant to the present discussion, since its imposition had little influence on the calculated permeability.) In order to lend further support to this hypothesis, we will study the Reynolds equation for a fracture geomety that is simple enough to allow analytical treatment, but which still captures some of the characteristics of "rough-walled" fractures. To accomplish this, we first restrict eq. (2) to one dimension. Although this is an approximation, note that the flow will always be locally one-dimensional on a small enough scale; furhermore, the two-dimensional character of the flow field will be accounted for by an averaging procedure. If the $x$ axis is chosen so as to coincide with the macroscopic pressure gradient, then the onedimensional version of (2) is simply

$$
\frac{d}{d x}\left[d^{3}(x) \frac{d p}{d x}\right]=0 .
$$

A single integration of (5) yields

$$
d^{3}(x) \frac{d P}{d x}=C,
$$


where $C$ is a constant of integration. Comparison of (3) and (6) shows that the constant of integration is merely $12 \mu \mathrm{Q}$, where $\mathrm{Q}$ is the volumetric flow rate. A second integration yields

$$
P_{2}-P_{1}=12 \mu Q \int_{x_{1}}^{x_{2}} \frac{d x}{d^{3}(x)}
$$

This result expresses the pressure drop between two points $x_{1}$ and $x_{2}$ in terms of a certain integral of the aperture function $d(x)$. If $(7)$ is expressed in terms of the hydraulic aperture $d_{h}$, the result is

$$
Q=\frac{d_{h}^{3}}{12 \mu} \frac{\Delta P}{L} \text {, where } d_{h}=\left[\frac{1}{L} \int_{x_{1}}^{x_{2}} \frac{d x}{d^{3}(x)}\right]^{-1 / 3} \text {, }
$$

and $L=x_{2}-x_{1}$. Using brackets to denote the "mean value", (8) can be expressed" as $d_{h}=\left\langle d^{-3}\right\rangle^{-1 \beta}$. It is worth noting that although (8) was derived as a solution of the approximate Reynolds equation, it can also be cierived ${ }^{10,11}$ as a rigorous "first approximation" to the full Navier-Stokes equations.

\section{SINUSOIDAL APERTURE VARIATION MODEL}

One of the simplest aperture profile functions that captures some of the geometrical properties of a "rough-walled" fracture is a constant aperture fracture with a sinusoidal penurbation (Fig. 2):

$$
d(x)=d_{m}[1+\delta \sin (2 \pi x / \lambda)] \text {. }
$$


where $d_{m}$ is the mean aperture, $\delta$ is the magnitude of the "roughness", and $\lambda$ is the wavelength of the aperture oscillations. For now, we imagine that all cross-sections parallel to the plane of Fig. 2 are identical. If the flow is in the direction transverse to the apenure oscillations (i.e., the $\mathrm{x}$ direction), then the hydraulic aperture can be found by considering ( 8 ) over one period of the oscillation:

$$
d_{h}{ }^{-3}=\frac{1}{\lambda} \int_{0}^{\lambda} \frac{d x}{d_{m}^{3}(1+\delta \sin 2 \pi x / \lambda)^{3}} .
$$

A simple change of variables, $\zeta=2 \pi \times / \lambda$, reduces $(10)$ to

$$
\mathrm{d}_{\mathrm{h}}{ }^{-3}=\frac{1}{2 \pi} \int_{0}^{2 \pi} \frac{\mathrm{d} \zeta}{\mathrm{d}_{m}^{3}(1+\delta \sin \zeta)^{3}},
$$

which shows that, within the framework of the lubrication approximation, the wavelength of the roughness does not affect the hydraulic aperture. (The wavelength does enter into higher-order approximations to the Navier-Stokes equations, as might be expected. ${ }^{11}$ ) Evajuation of (11) gives ${ }^{12}$

$$
d_{h}^{3}=d_{m}{ }^{3} \frac{\left(1-\delta^{2}\right)^{5 / 2}}{1+\left(\delta^{2} / 2\right)}
$$

The above one-dimensional model assumes, in a sense, that the resistances due to each aperture element are in series, since the flow through each element is the same. If each aperture $d$ is thought of as having a resistance proportional to $d^{-3}$, then (7) corresponds to all of the resistances being placed in series. The other "extreme" 
assumption would be that all of the resistances are in parallel. This would correspond to flow perpendicular to the plane of Fig. 2, and would be equivalent to an hydraulic aperture given by $d_{h}^{3}=\left\langle d^{3}\right\rangle$, i.e., ${ }^{13}$

$$
\begin{aligned}
d_{h}^{3} & =\frac{1}{\lambda} \int_{0}^{\lambda} d^{3}(x) d x \\
& =\frac{1}{2 \pi} \int_{0}^{2 \pi} d_{m}^{3}(1+\delta \sin \zeta)^{3} d \zeta \\
& =d_{m}^{3}\left[1+\left(3 \delta^{2} / 2\right)\right] .
\end{aligned}
$$

Note that while (12) shows that $d_{h}<d_{m}$ for flow transverse to the roughness, (13) indicates that $d_{h}>d_{m}$ for flow parallel to the roughness.

It is known from network theory ${ }^{14}$ that the assumption that all of the resistors in a random resistor network are in series (or parallel) provides lower (or upper) bounds respectively to the actual effective conductivity. In our problem, we know that at some points the fluid will be flowing parallel to the aperture oscillations, while in some cases it will be flowing transverse to the oscillations. One simple way to arrive at an estimate of the effective conductivity of a "random mixture" of these two cases is to use the geomerric mean of the conductivities given by (12) and (13):

$$
d_{h}^{3}=\sqrt{d_{h}^{3}(\text { series }) \times d_{h}^{3}(\text { parallel })}=\sqrt{\left\langle d^{-3}\right\rangle^{-1}\left\langle d^{3}\right\rangle}
$$




$$
=\mathrm{d}_{\mathrm{m}}^{3}\left\{\frac{\left[1+\left(3 \delta^{2} / 2\right)\right]\left(1-\delta^{2}\right)^{5 / 2}}{1+\left(\delta^{2} / 2\right)}\right\}^{1 / 2} .
$$

A somewhat more sophisticated estimation of the effective conductivity can be found by appealing to the upper and lower bounds that were derived by Hashin and Shtrik$\operatorname{man}^{15}$ for the effective conductivity of a sandom mixture consisting of two "components" with different conductivities. These bounds are known to be closer together than the above-mentioned series and parallel bounds. For a 50-50 mixture of elements with cunductivities $G_{1}$ and $G_{2}$, where $G_{1}<G_{2}$, the Hashin-Shtrikman bounds on the overall conductivity are

$$
G_{1}+\frac{3 G_{1}\left(G_{2}-G_{1}\right)}{5 G_{1}+G_{2}}<G<G_{2}-\frac{3 G_{2}\left(G_{2}-G_{1}\right)}{5 G_{2}+G_{1}}
$$

Aside from the factor of $1 / 12 \mu$, which is common to all terms, the hydraulic conductance is equivalent to the cube of the hydraulic aperture, i.e., $G=d_{h}^{3} / 12 \mu$. Hence the bounds (15) can be applied to $d_{h}^{3}$, with (12) and (13) used for $G_{1}$ and $G_{2}$. The geometric mean of these two bounds will provide an estimate of the effective conductance that has an narrower possible margin of error than the geometric mean of the series and parallel bounds, given by (14).

In order to compare our semi-analytical results with those of Brown ${ }^{8}$ or Patir anc Cheng, ${ }^{3}$ we also need an expression for the standard deviation of the height distribution, $\sigma$. Since $d-d_{m}=d_{m} \delta \sin (2 \pi \times / \lambda)$,

$$
\sigma=\left[\frac{1}{2 \pi} \int_{0}^{2 \pi} \mathrm{d}_{\mathrm{m}}^{2} \delta^{2} \sin ^{2} \zeta \mathrm{d} \zeta\right]^{1 / 2}=\mathrm{d}_{\mathrm{m}} \delta \sqrt{2}
$$


Hence $\delta=\sqrt{2} \sigma / \mathrm{d}_{\mathrm{m}}$ for the sinusoidal profile shown in Fig. 2, and using this fact we can plot the geometric mean from expression (14), and the mean of the HashinShrrikman bounds from (15), alongside the results of Brown ${ }^{8}$ and Patir and Cheng. ${ }^{3}$ This is done in Fig. 3, where it is seen that all of the results are qualitatively similar, showing appreciable divergence only for small values of $d_{m} / \sigma$ (i.e., very rough surfaces). The simple geometric mean of the series and parallel conductivities yields almost the same result as the geometric mean of the Hashin-Shtrikman upper and lower bounds. (This lends further credence to the use of the geometric mean to estimate effective permeabilities. In the somewhat different context of two-dimensional porous formations with stochastic permeability distributions, Dagan ${ }^{16}$ showed that the geometric mean often yields an accurate estimate of the effective permeability). Each of these means matches the curve of Patis and Cheng closely for $d_{m} / \sigma>3$, but fall below this curve for smaller values of $\mathrm{d}_{\mathrm{m}} / \sigma$. The data of $B$ rown ${ }^{8}$ fall close to the curve of Patir and Cheng, ${ }^{3}$ although these data approach the asymptotic value of $d_{h} / d_{1 n}=1$ more slowly as $d_{m} / \sigma \rightarrow \infty$.

Fig. 4 shows the geometric mean of the series and parallel bounds for the sinusoidal model, compared with the upper and lower values of each set of ten realizations considered by Brown at each value of $d_{m} / \sigma$. This geometric mean generally lies within the range of values found by Brown; it falls near the upper range for large values of $\mathrm{d}_{m} / \sigma$, and towards the lower range for small values of $\mathrm{d}_{m} / \sigma$. For smali values of $d_{m} / \sigma$, which is to say reianvely rough surfaces, the sinusoidal model permeabilities drop off more rapidly than do the mean values found by either $\mathrm{Brown}^{8}$ or Patir and Cheng. ${ }^{3}$ This is easily understood because, for example, as $\delta \rightarrow 1$, the "series" conductivity goes to zero, and so both sets of lower bounds upon which the "geometric means" are based will vanish. Since $d_{m} / \sigma=\sqrt{2} / \delta$, this occurs at $d_{m} / \sigma=1.414$. In reality, of course, the flow would simply flow around any local point of contact between the two faces of the fracture, and the hydraulic conductivity would 
not drop to zero. This possibility is not entirely accounted for by the nvera.ging methods that we have used to find the overall effective permeability.

It is borthwhile to investigate to what evtent additional soughness with higher spatial frequencies alters the analytical results This can be done by using a prosile that contains 'wo sinusoidal components (see Fig. ju:

$$
d(x)=d_{m}\left[1+\delta_{1} \sin \left(2 \pi x / \lambda_{1}\right)+\delta_{2} \sin \left(2 \pi x / \lambda_{2}\right)\right] .
$$

In this case an analytical evaluation of $\left\langle\mathrm{d}^{3}\right\rangle,\left\langle\mathrm{d}^{-3}\right\rangle$, and $\sigma$ is not practical, but can be easily carried out numerically. In general, the results show that the addition of this "smaller scale roughness" has only a minor effect on the relationship between $d_{h} / d_{\mathfrak{m}}$ and $d_{m} / \sigma$. As an example, consider the addition of a roughness component with a wavelength of one-half of the dominant wavelength, i.e., $\lambda_{1} \lambda_{2}=2$. A redsonable value for the amplituae of this component can be found by the following consideratioris. The power spectral density of a rock surface is usually of a form that can be expressed as $^{17}$

$$
\delta(\lambda)=\underset{:}{\text { constant }} \times \lambda^{3.5-D}
$$

where $2<D<3$ is the fractal dimension of the surface. Most rock surfaces seem to have fractal dimensions that lie between 2 and 2.5, since fractal dimensions near 3 correspond to an extreme degree of roughness, for which nearby apertures are completely uncorrelated. ${ }^{18}$ Using a value of $\mathrm{D}=2.5$, as in those results of $\mathrm{Brown}{ }^{8}$ plotted on Fig. 3, we see that $\delta$ is proporional to $\lambda$, and so reasonable values for $\delta_{2}$ can be found by setring $S_{2} / \delta_{1}=0.50$. Note that the ptrpose of this calculation is merely to find values of $\delta_{2}$ that can be used as a meaningful example, and is not intended to be 
"exact" in any way. Fig. 6 shows the geometric mean of the series and parallel conductances for this model, compared with the results for $\delta_{2}=0$. The addition of a higher-frequency roughness component is seen to have little effect on the $d_{h} / d_{m}$ vs. $\mathrm{d}_{\mathrm{m}} / \sigma$ relationship. We have tested this result by adding further roughness components of smaller wavelength, and this conclusion seems to hold in general.

In order to further estimate the sensitivity of the analytical result (14b) to the shape of the aperture profile, a similar analysis has been caried out for a fracture with a "sawtooth" profile, such as that used by Elsworth and Goodman. 19 The extreme case, as far as irregularity of the aperture distribution is concerned, is when one face of the fracture is displaced from the other by one-half of a wavelength (see Fig. 11 of Elsworth and Goodman ${ }^{19}$ ). Within a "unit cell" consisting of one-half a wavelength, the aperture can be expressed as $d(x)=d_{\min }+\left(d_{\max }-d_{\min }\right) x / L$. The various statistical parameters can readily be found to be $d_{m}=\left(d_{\max }-d_{\min }\right) / 2, \sigma=\left(d_{\max }-d_{\min }\right) / 2 \sqrt{3}$, $\left\langle\mathrm{d}^{3}\right\rangle=\mathrm{d}_{\mathrm{m}}^{3}+3 \mathrm{~d}_{\mathrm{m}} \sigma^{2}$, and $\left\langle\mathrm{d}^{-3}\right\rangle^{-1}=\left(\mathrm{d}_{\mathrm{m}}^{2}-3 \sigma^{2}\right)^{2} / \mathrm{d}_{\mathrm{m}}$. The geometric mean of the series and parallel conductances, which in general is is given by (14a), takes the form

$$
d_{h}^{3}[\text { sawtooth }]=d_{m}^{3}\left\{\left[1-9\left(\sigma / d_{m}\right)^{4}\right]\left[1-3\left(\sigma / d_{m}\right)^{2}\right]\right\}^{1 / 2}
$$

This is a different relationship between $d_{h} / d_{m}$ and $\sigma / d_{m}$ than was predicted for the sinusoidal profile. However, when plotted as in Fig. 3, (19) lies very close to (14b); in fact, the two expressions agree exactly to "first order": $\left(\mathrm{d}_{\mathrm{h}} / \mathrm{d}_{\mathrm{m}}\right)^{3}=1-1.5\left(\sigma / \mathrm{d}_{\mathrm{m}}\right)^{2}$. Within the context of our analytical model, therefore, the dependence of the normalized permeability on the single parameter $\sigma / d_{m}$ appears to be somewhat insensitive to shape. 
The results discussed above, and shown in Figs, 3 and 6, lead us to conclude that within the context of the lubrication approximation there is a strong comelation between the parameters $d_{h} / d_{m}$ and $d_{m} / \sigma$, with the additional statistical details of the surface roughness profile providing only a small perturbation on this relationship. The fact that our quasi-analytical results are similar to the numerical results found by Patir and Cheng ${ }^{3}$ and Brown ${ }^{8}$ lends support to this conjecture. However, all of these analyses are predicated on the use of the lubrication approximation to reduce the full Navier-Stokes equations to the Reynolds equation. We now carry out some analysis aimed at estimating the errors incurred by using the lubrication approximation, and at delineating those ranges of the fracture roughness parameters that will allow this approximation to be used.

\section{HIGHER-ORDER CORRECTIONS}

One of the assumptions needed to justify the reduction of the Navier-Stokes equations to the Reynolds equation is that the velocity gradients in the plane of the fracture are much smaller than the velocity gradient in the direction perpendicular to the fracture plane. Brown ${ }^{2}$ correctly states that this is in some sense equivalent to the condition (in the present notation) that $\sigma / \lambda<1$; this can be understood by noting that rapid changes in the aperture will necessitate rapid variations in the in-plane velocity, in order to maintain conservation of mass. Interestingly enough, this condition imposes no restrirtions on the ratio $\sigma / d_{m}$; ;elatively large values of $\checkmark$ are permissable, as long as the variation in aperture occurs slowly in the $x$ or $y$ directions. Brown ${ }^{2}$ examined the velocity gradients that he computed numerically, and found that the condition on their magnitudes was not always satisfied. In fact, he concluded that the fracture walls would have to be smonth on length scales on the order of $500-5000 \mu \mathrm{m}_{r}$ which will not always be the case for real fractures. ${ }^{18}$ (Note that Fig. 3 implies that a fiacture can be considered "hydraulically" smooth if the amplitude of any roughness is less than 
about $0.1 \mathrm{~d}_{\mathrm{m}}$ ). Furthermore, Brown's analysis does not quantify the errors that are incurred by use of the lubrication approximation for fractures with "rapidly-varying" apertures.

Strict "error estimates" for the lubrication theory approximation to the NavierStokes equations are difficult to derive. A more tractable approach is to focus on a specific geometry such as that shown in Fig. 2, and examine the solutions to higherorder approximations to the Navier-Stokes squations. In this way we can find the range of values for the parameter $\sigma \lambda$ for which the lubrication approximation is "valid". This will provide us with a rough rule-of-thumb that should apply to more general fracture aperture profiles.

Hasegawa and Izuchi ${ }^{11}$ performed a perturuation analysis of the problem of flow between a smooth wall and a wall with sinusoidal roughness. Although this geometry is slightly different from that shown in Fig. 2, it can still serve to demonstrate th: influence of the effects of roughness, wavelength, etc., oit permeability. Their results can be put into a form in which the small perturbation parameters are $\sigma / \lambda$ and $R e$, the Reynolds number. We will set $\operatorname{Re}=0$ in their expansions, and concentrate on the effect of $\sigma / \lambda$. Note that these authors fixed the pressure gradient along the channel, and found perturbation expansions for the volumetric flow rate; their results are therefore more readily usable, for our purposes, than the related results of Van Dyke, ${ }^{20}$ who considered a fixed flow rate, and found expressions for the stream function.

When translated into the present notation, the second-order expansion found by Hasegawa and Izuchi ${ }^{11}$ can be expressed as (see their eqs. (25) and (27), and Fig. 1)

$$
d_{h}^{3}=d_{m}^{3} \frac{\left(1-\delta^{2}\right)^{5 / 2}}{1+\left(\delta^{2} / 2\right)}\left[1-\frac{6 \pi^{2}\left(1-\delta^{2}\right) \delta^{4}}{10\left[1+\left(\delta^{2} / 2\right)\right]}\left[\frac{d_{m}}{\lambda}\right)^{2}\right]
$$


Comparison of (20) and (12) shows that the second term in brackets in (20) is the correction due to nonzero values of $\sigma / \lambda$. At first sight it might appear that, due to the $1 / \lambda^{2}$ dependence, the correction term could easily be very large if $\lambda$ were small enough. However, the amplitude of the roughness usually drops off rapidly with increasing spatial frequency. For example, (18) implies that as $\lambda$ decreases, $\delta$ also decreases, because $\delta=$ constant $\times \lambda^{s}$. As indicated above, realistic values of $s$ lie between 1 and 1.5 (c.mesponding to surface fractal dimensions between 2 and 2.5). Hence we see that if $\lambda$ is small, $\delta$ will necessarily be small also, and the correction term will remain bounded. For example, consider the "worst case", $s=1$, for which $\delta=C \lambda$. The correction term in (20) then scales as $\lambda^{4} / \lambda^{2}=\lambda^{2}$, and will therefore be very small for small spatial wavelengths. This is analogous to the fact, well-known to fluid-flow engineers, that for laminar flow in a pipe, small-scale roughness has no effect on the hydraulic resistance. This insensitivity of laminar pipe flow to smallscale roughness is illustrated in the "Moody-Nikuradse" charts, ${ }^{4}$ in which the "friction factor" is plotted against the Reynolds number. The curves corresponding to different values of the "relative roughness" do not diverge until the turbulent (high Reynolds number) regime.

The most stringent condition that we can derive by requiring the correction term to be small is actually found by considering the longest wavelength roughness. We first use (16) to replace $d_{m}$ with $\sqrt{2} \sigma / \delta$, and note that, over the range of definition of $\delta$, which is $0<\delta<1$, the maximum value of the term that multiplies $(\sigma / \lambda)^{2}$ is 2.39 . Therefore, if we want to restrict the relative magnitude of the correction term to $10 \%$ of the value predicted by lubrication theory, we must have $2.39(\sigma \lambda)^{2}<0.10$, which implies $\lambda>5 \sigma$. This condition is much less restrictive than the condition that was postulated by Brown, ${ }^{2}$ which was $\lambda>50 \sigma$. If we agree that the aperture of a fracture such as shown in Fig. 2 can be considered nearly constant over lengths not greater than about $\lambda / 10$, say, this new criterion can (very roughly) be viewed as requiring the 
surfaces to be nearly constant over lengths on the order of $\sigma$. Further evidence supporting this conclusion can be found in the work of Pozrikidis, ${ }^{21}$ who used a boundary-integral method to study the same problem that Hasegawa and Izuchi ${ }^{11}$ analyzed by perturbation methods. Although Pozrikidis did not solve for the flow rate, he did show that as long as $\lambda>5 \sigma$, the streamlines will adjust to the curvature of the wall, and no eddies will be generated within the sinusoidal bumps. Since the existence of such eddies would caust a breakdown of the quasi-one-dimensional lubrication assumption, the results of Pozrikidis corroborate our conclusion that the lubrication assumption will not become invalid as long as $\lambda>5 \sigma$.

\section{SUMMARY AND CONCLUSIONS}

The lubrication approximation has been used to study the permeability of roughwalled rock iractures. A major purpose of this study was to develop an understanding of how the hydraulic aperture depends on the statistics of the aperture distribution. When the various aperture elements are in series, the effective hydraulic aperture is given by $d_{h}=\left\langle d^{-3}\right\rangle^{-1 / 3}<d_{\text {mean }}$ while if they are arranged in parallel, $\left.d_{h}=\left\langle d^{3}\right\rangle^{1 / 3}\right\rangle d_{\text {mean }}$. Since the assumption of series (or parallel) resistances vastly underestimates (or overestimates) the actual effective conductance, ${ }^{14}$ a further averaging of these two values is needed. This can be achieved using either the geometric mean of the series and parallel conductances, or the geometric mean of the (narrower) Hashin-Shrrikman bounds; both methods provide similar estimates of the effective hydraulic apernure.

For fractures whose apertures vary sinusoidally (or in a sawtooth manner), analytical expressions were found for $\left\langle d^{3}\right\rangle$, and $\left\langle d^{-3}\right\rangle$, in terms of $d_{m}=\langle d\rangle$, and the standard deviation of the aperture, $\sigma$, which is equal to $\sqrt{\left\langle\left(d-d_{m}\right)^{2}\right\rangle}$. The motivation for analyzing these models was that they permit analytical treatment, while still capturing some of the qualities of "roughness". For these models, the predicted hydraulic 
aperture is always less than the mean aperture, by an amount that increases with increasing roughness. In fact, the resulting relationships between $d_{h} / d_{m}$ and $\sigma / d_{m}$ are very similar to that found numerically by Brown ${ }^{8}$ and Patir and Cheng, ${ }^{3}$ who studied fractures with highly irregular roughness profiles. Our analytical expression for $d_{h}$ for the sinusoidal model fell within the spread of values found by Brown in his various stochastic realizations.

An attempt was also made to estimate the conditions under which the lubricaticn approximation would be valid in treating flow through fractures. Although this approximation has been frequently used, quantitative estimates of its accuracy have not been available. By examining the higher-order solutions to the Navier-Stokes equations for flow through a sinusoidally-varying channel developed by Hasegawa and Izuchi, ${ }^{11}$ we have been able to make some comments regarding this question. Deviations from the permeability predicted under the lubrication approximation seem to become appreciable only when the spatial wavelength of the dominant roughness component becomes on the order of (or smaller than) the amplitude of that roughness. This implies that the condition hypothesized by Brown, ${ }^{2}$ which was that the fracture should be of nearly constant aperture over distances of at least $50 \sigma$, was in fact too conservaive. If our conclusion is correct, then the use of the lubrication approximation would be justifed for many real fractures, and the more difficult Navier-Stokes analysis could be avoided.

\section{ACKNOWLEDGMENTS}

This work was done under U.S. Department of Energy Contract No. DE-AC0376SF00098, administered by the Nevada Operations Office, in cooperation with the U.S. Geological Survey, Denver. The authors thank Carol Boughton and Barney Lewis of the USGS for supporting this work, and thank C. H. Lai and Y. W. Tsang of LBL, and E. Kwicklis and T. Brady of the USGS, for reviewing this paper. 


\section{REFERENCES}

1. J. B. WALSH, "Effect of pore pressure and confining pressure on fracture permeability," Int. J. Rock Mech. Min. Sci. \& Geomech. Abstr., 18(5), 429 (1981).

2. S. R. BROWN, "Fluid flow through rock joints: the effect of surface roughness," J. Geophys. Res., 92(B2), 1337 (1987).

3. N. PATIR and H. S. CHENG, "An average flow model for determining effects of three-dimensional roughness on partial hydrodynamic lubrication," ASME J. Lubr. Technol., 100(1), 12 (1978).

4. H. SCHLICHTING, Boundary-Layer Theory, 6th Ed., Mc-Graw-Hill, New York (1968).

5. W. F. HUGHES, Introduction to Viscous Flow, McGraw-Hill, New York (1979).

6. D. T. SNOW, "Rock fracture spacings, openings, and porosities," J. Soil Mech. Found. Div. ASCE, 94(SMI), 73 (1968).

7. F. M. MILNE-THOMSON, Theoretical Hydrodynamics, 5th Ed., Macmillan, New York (1968),

8. S. R. BROWN, "Transport of fluid and electric current through a singie fracture," J. Geophys. Res., 94(B7), 9429 (1989).

9. S. E. SILIMAN, "An interpretation of the difference between aperture estimates derived from hydraulic and tracer tests in a single fracture," Water Resour. Res., $25(10), 2275$ (1989).

10. W. E. LANGLOIS, Slow Viscous Flow, Macmillan, New York (1964). 
11. E. HASEGAWA and H. ZZUCHI, "On steady flow through a channel consisting of an uneven wall and a plane wall, Part 1, Case of no relative motion in two walls," Bull. Jop. Soc. Mech. Eng., 2б, 514 (1983).

12. I. S. GRADSHTEYN and I. M. RHYZIK, Table of Integrals, Series, and Products, Academic Press, San Diego (1980).

13. C. E. NEUZIL and J. V. TRACY, "Flow through fractures," Waser Resour. Res., 17(1), 191 (1981).

14. T. R. MADDEN, "Random networks and mixing laws," Geophysics, 4I(6A), 1104 (1976).

15. Z. HASHIN and S. SHTRIKMAN, "A variational approach to the theory of the effective magnetic permeability of multiphase materials," J. Appl. Phys., 33(I0), 3125 (1962).

16. G. DAGAN, "Models of groundwater flow in statistically homogeneous porous fo. mations," Water Resour. Res., 15(1), 47 (1979).

17. S. KUMAR and G. S. BODVARSSON, "Fractal study and simulation of fracture roughness," Geophys, Res. Letts., 17(6), 701 (1990).

18. S. R. BROWN and C. H. SCHOLZ, "Broad bandwidth study of the topography of natural rock surfaces," J. Geophys. Res., 9O(B14), 12575 (1985).

19. D. ELSWORTH and R. E. GOODMAN, "Characterization of rock fissure hydraulic conductivity using idealized wall roughness profiles," Int. J. Rock Mech. Min. Sci. \& Geomech. Absir., 23(3), 233 (1986).

20. M. VAN DYKE, "Slow variations in continuum mechanics," Adv. Appl. Mech., 25,1 (1987). 
21. C. POZRIKIDIS, "Creeping flow in two-dimensional channels," J. Fluid Mech., 180, 495 (1987).

22. K. G. RAVEN, K. S. NOVAKOWSKI, and P. A. LAPCEVIC, "Interpretation of field tracer tests of a single fracture using a transient solute storage model, "Water Resour. Res., 24(12), 2019 (1988). 


\section{FIGURE CAPTIONS}

Figure 1. Plot showing the effect of roughness on permeability. The hydraulic aperture is $d_{h}$, the mean aperture is $d_{m}$, and the standard deviation of the aperture is $\sigma$. The curve labelled "Patir and Cheng" is from (4), which was fit by them to their numerical results, while the data points labelled "Brown" each represent the mean of ten different realizations of surfaces with fractal dimensions of $\mathbf{2 . 5}$.

Figure 2. Fracture with a sinusoidal variation in aperture. $d_{m}$ is the mean aperture, $\delta$ is the (relative) amplitude of the aperture variation, and $\lambda$ is the wavelength of the aperture variation.

Figlire 3. Normalized permeability of a fracture as a function of the standard deviation of the roughness. Two different averaging methods, using the series/parallel bounds and the Hashin-Shtrikman bounds, have been used for the sinusoidal model.

Figure 4. Normalized permeability of a fracture as a function of the standard deviation of the roughness. The geometric mean of the series and parallel bounds for the sinusoidal model is compared to the range of values computed by Brown [2] for a fractal dimension of 2.5 .

Figure 5. Fracture whose aperture variation contains two sinusoidal components, as represented by (17), with $\lambda_{1} / \lambda_{2}=2, \delta_{1} / \delta_{2}=2$.

Figure 6. Comparison of the permeabilities of fractures with one or two sinusoidal components in their aperture variation (see Figures 1 and 5), according to the geometric mean of the series and parallel values. The ratio $\lambda_{1} / d_{m}$ is varied, while maintaining $\lambda_{1} / \lambda_{2}=2$ and $\delta_{1} / \delta_{2}=2$. 


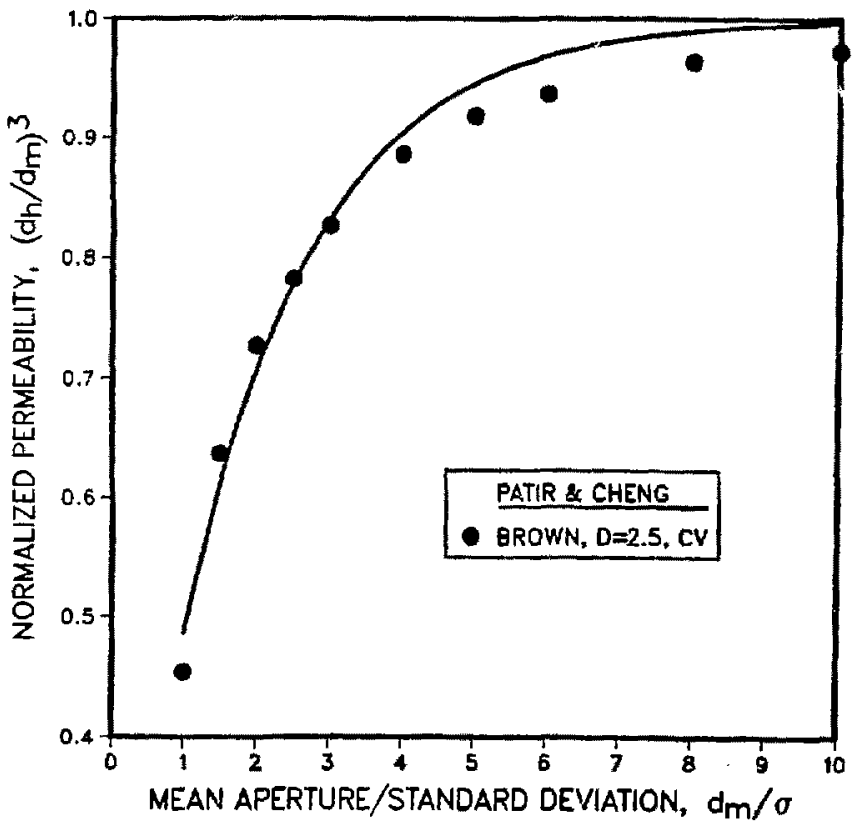

Figure 1. Plot showing the effect of roughness on permeability. The hydraulic aperture is $d_{h}$, the mean aperture is $d_{m}$, and the standard deviation of the aperture is $\sigma$. The curve labelled "Patir and Cheng" is from (4), which was fit by them to their numerical results, while the data points labelled "Brown" each represent the mean of ten different realizations of surfaces with fractal dimensions of 2.5 . 


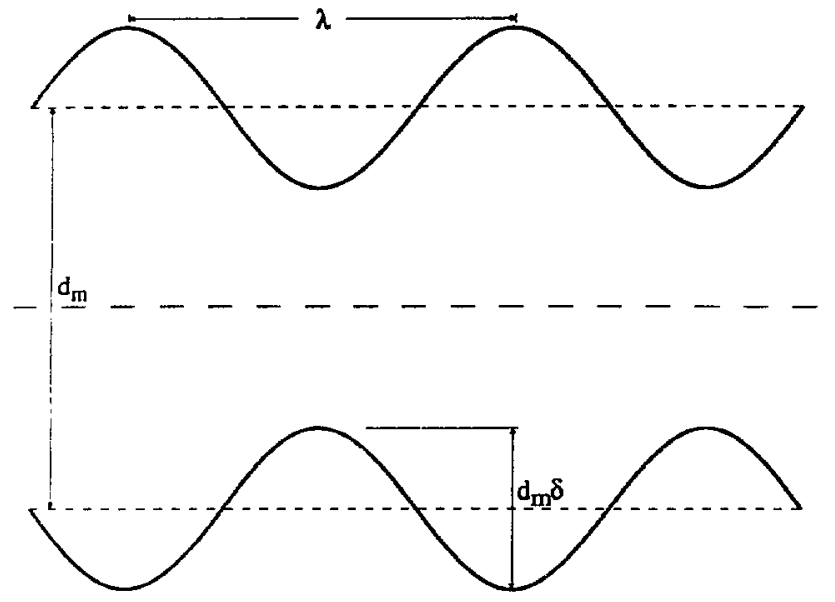

ESD-9010-0050

Figure 2. Fracture with a sinusoidal variation in aperure. $d_{m}$ is the mean aperture, $\delta$ is the (relative) amplitude of the anerture variation, and $\lambda$ is the wavelength of the aperture variation. 


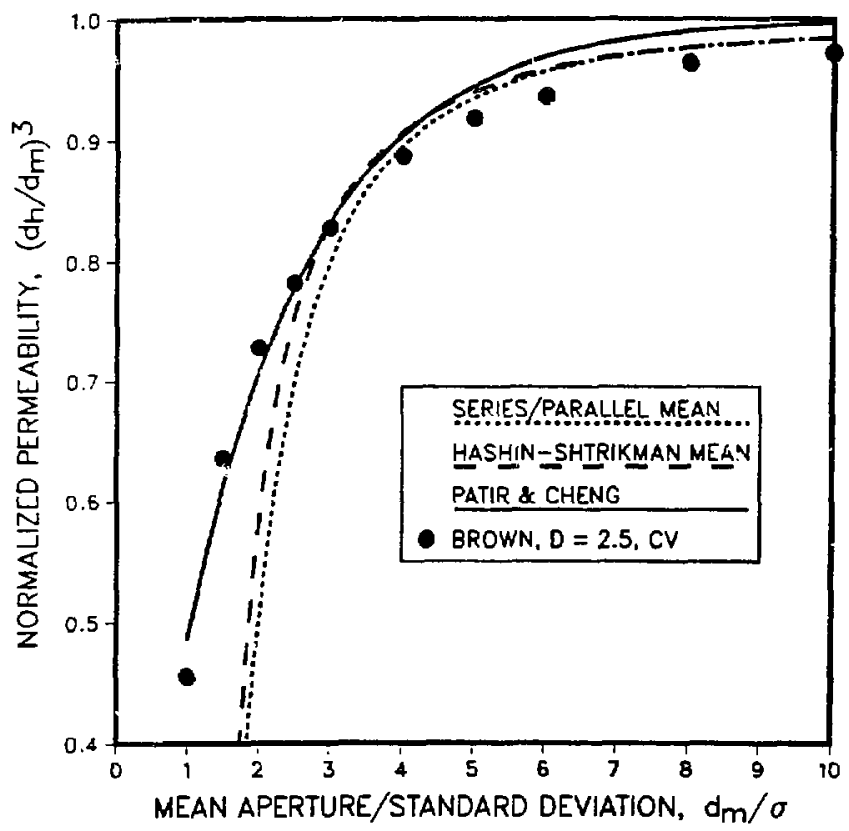

Figure 3. Normalized permeability of a fracture as a function of the standand deviation of the roughness. Two different averaging methods, using the series/parallel bounds and the Hashin-Shtrikman bounds, have been used for the sinusoidal model. 


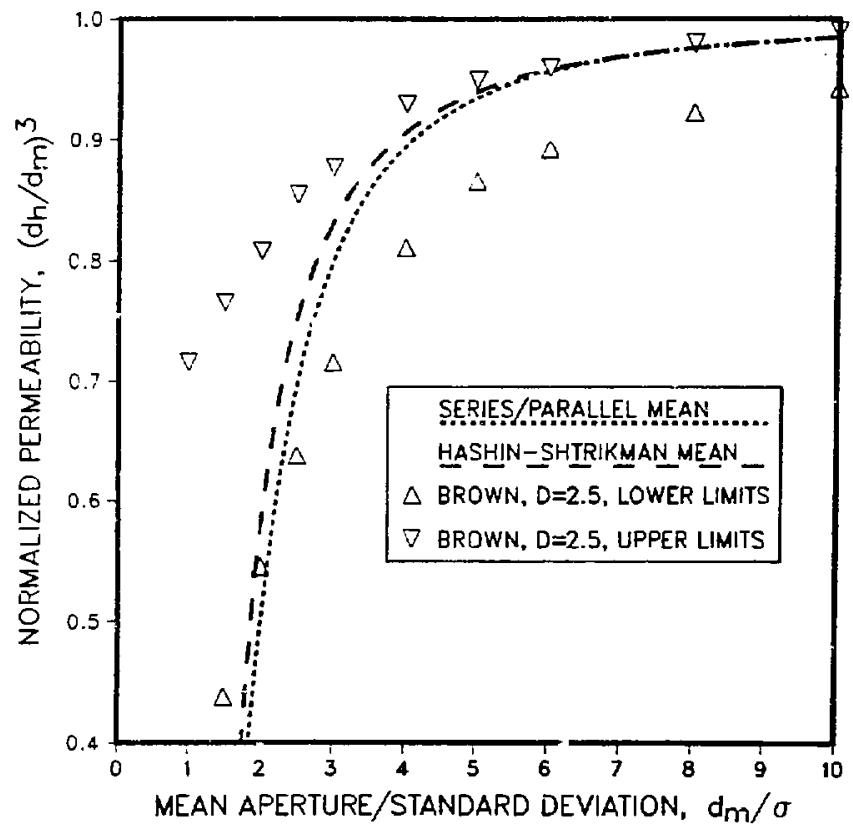

Figure 4. Normalized permeability of a fracture as a function of the standard deviation of the roughness. The geometric mean of the series and parallel bounds for the sinusoidal model is compared to the range of values computed by Brown [2] for a fractal dimension of 2.5 . 


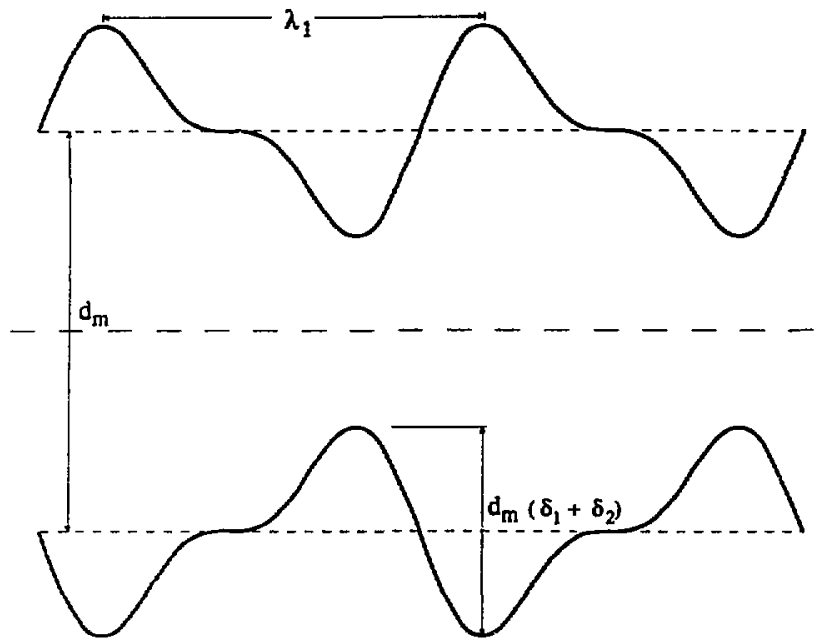

ESD-9010-0051

Figure 5. Fracture whose aperture variation contains two sinusoidai components, as repre:ented by (17), with $\lambda_{1} / \lambda_{2}=2, \delta_{1} / \delta_{2}=2$. 


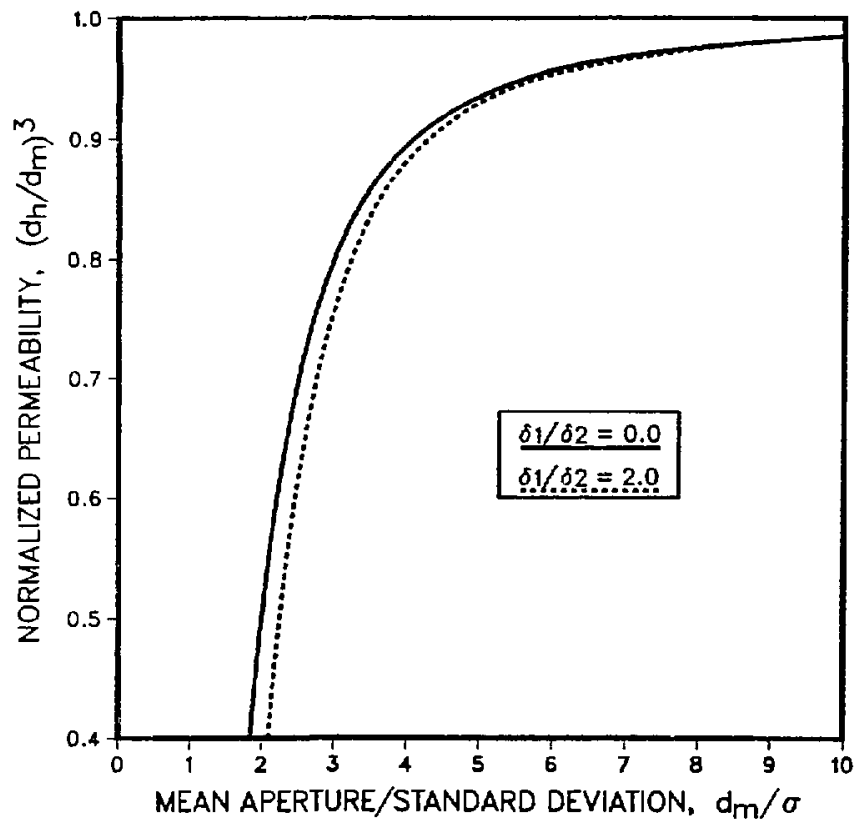

Figure 6. Comparison of the permeabilities of fractures with one or two sinusoidal components in their apenure variation (see Figures 1 and 5), accorcing to the geometric mean of the series and parallel values. The ratio $\lambda_{1} / d_{m}$ is varied, while maintaining $\lambda_{1} / \lambda_{2}=2$ and $\delta_{1} / \delta_{2}=2$. 